ANL-6747

Chemistry

(TID-4500, 21 st Ed.)

AEC Research and

Development Report

ARGONNE NATIONAL LABORATORY

9700 South Cass Avenue

Argonne, Illinois 60440

TAB LES OF OXYGEN PRESSURES CALCULATED

FROM THE HYDROGEN-WATER SYSTEM AND

FROM THE DISSOCIATION OF CUPRIC OXIDE

by

L. M. Atlas and J. B. Moser

Metallurgy Division

Program 9.6.5

July 1963

Operated by The University of Chicago

under

Contract W-31-109-eng-38

with the

U.S. Atomic Energy Commission 


\section{DISCLAIMER}

This report was prepared as an account of work sponsored by an agency of the United States Government. Neither the United States Government nor any agency Thereof, nor any of their employees, makes any warranty, express or implied, or assumes any legal liability or responsibility for the accuracy, completeness, or usefulness of any information, apparatus, product, or process disclosed, or represents that its use would not infringe privately owned rights. Reference herein to any specific commercial product, process, or service by trade name, trademark, manufacturer, or otherwise does not necessarily constitute or imply its endorsement, recommendation, or favoring by the United States Government or any agency thereof. The views and opinions of authors expressed herein do not necessarily state or reflect those of the United States Government or any agency thereof. 


\section{DISCLAIMER}

Portions of this document may be illegible in electronic image products. Images are produced from the best available original document. 
TABLE OF CONTENTS

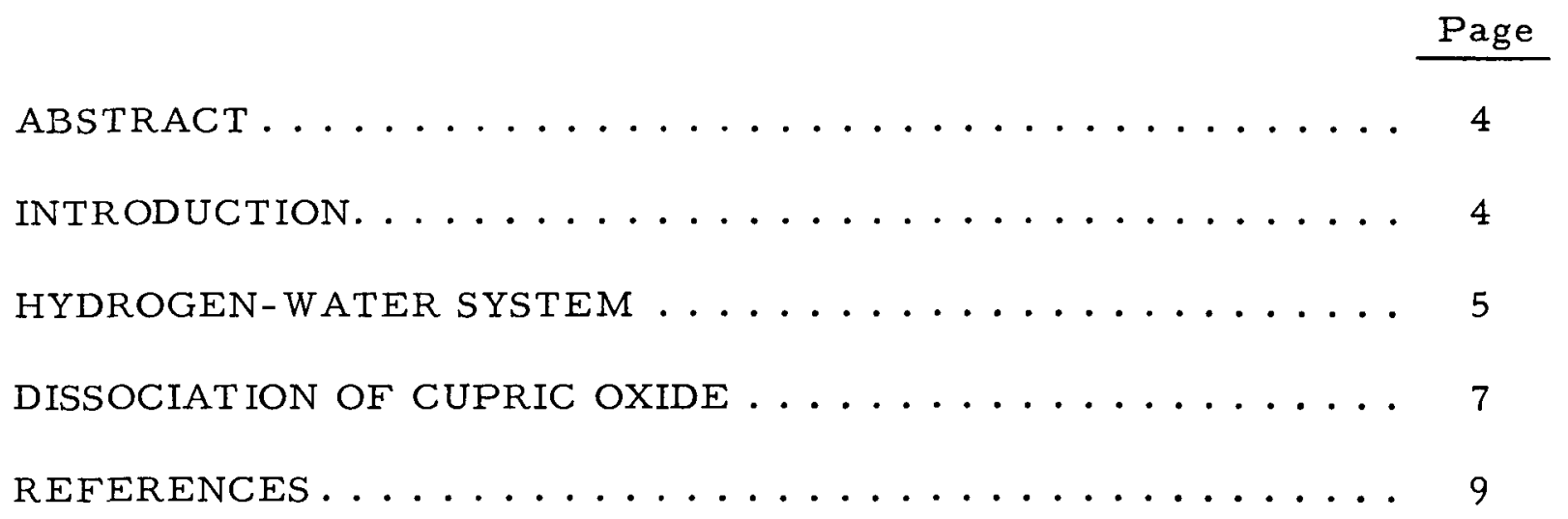




\section{LIST OF TABLES}

No.

Title

Page

I. Partial Pressure of Oxygen in Equilibrium with Hydrogen and Water Vapor ....................... 6,7

II. Dissociation Pressure of Oxygen from Cupric Oxide. . . . . 8 


\title{
TABLES OF OXYGEN PRESSURES CALCULATED \\ FROM THE HYDROGEN-WATER SYSTEM AND \\ FROM THE DISSOCIATION OF CUPRIC OXIDE
}

by

L. M. Atlas and J. B. Moser

\begin{abstract}
The dissociation of various mixtures of hydrogen and water vapor and of cupric oxide has been calculated over a wide range of temperatures (from 600 to $1700^{\circ} \mathrm{C}$ for the $\mathrm{H}_{2}-\mathrm{H}_{2} \mathrm{O}$ system, and from 200 to $1000^{\circ} \mathrm{C}$ for $\left.\mathrm{CuO}\right)$. The resulting partial pressures of oxygen are presented in tabular form.
\end{abstract}

\section{INTRODUCTION}

Investigations of oxidation-reduction equilibria and kinetics of oxide systems require a knowledge of the partial pressure of oxygen in the gas phase. It is a common practice for oxygen pressures to be established with the aid of the known standard free energies $\left(\Delta F^{\circ}\right)$ for such reactions as

$$
\begin{aligned}
& \mathrm{H}_{2}+1 / 2 \mathrm{O}_{2} \rightleftarrows \mathrm{H}_{2} \mathrm{O} \\
& \mathrm{Cu}_{2} \mathrm{O}+1 / 2 \mathrm{O}_{2} \rightleftarrows 2 \mathrm{CuO} \\
& \mathrm{CO}+1 / 2 \mathrm{O}_{2} \rightleftarrows \mathrm{CO}_{2} \\
& \mathrm{Fe}+1 / 2 \mathrm{O}_{2} \rightleftarrows \mathrm{FeO}
\end{aligned}
$$

Unfortunately, few, if any, investigators distribute the results of their calculations of oxygen pressure $\mathrm{P}\left(\mathrm{O}_{2}\right)$; consequently, this labor undoubtedly has been repeated many times. The present tabulation started out to be just such a private list; therefore, no attempt has been made to make it all inclusive. However, for the hydrogen-water system these tables do present a variation of $\mathrm{P}\left(\mathrm{O}_{2}\right)$ over several decades at any chosen reaction temperature; in addition, for the dissociation of cupric oxide they give oxygen pressures ranging from about $10^{-20}$ to $0.1 \mathrm{~atm}$. The hydrogen-water reaction has the advantage of acting in the manner of a buffered system, that is, adsorption or evolution of a small amount of oxygen by the walls of the reaction vessel will not grossly affect $\mathrm{P}\left(\mathrm{O}_{2}\right)$ because of the comparative excess of $\mathrm{H}_{2} \mathrm{O}$ and $\mathrm{H}_{2}$ in the atmosphere. The copper oxide reaction does not give this buffering action and, therefore, has doubtful utility at low oxygen 
pressures. At higher pressures, however, above perhaps $10^{-8}$ or $10^{-7} \mathrm{~atm}$, the cupric-cuprous oxide system has the advantage of allowing $\mathrm{P}\left(\mathrm{O}_{2}\right)$ to be very simply regulated by controlling a furnace whose temperature is independent of that of the reaction being studied. Moreover, use of the copper oxide dissociation eliminates the possibility of unwanted side reactions, such as the formation of hydrides or hydroxides.

Whenever oxygen-containing atmospheres derived from either the $\mathrm{H}_{2}-\mathrm{H}_{2} \mathrm{O}$ or the $\mathrm{Cu}_{2} \mathrm{O}-\mathrm{CuO}$ systems are used to study an oxidation or reduction reaction, care should be taken that the reaction does not inadvertently change the partial pressure of oxygen of the gas phase. In general, the volume of gas should be large enough to act as an infinite reservoir as compared to the quantity of oxygen involved in the reaction.

\section{HYDROGEN-WATER SYSTEM}

Oxygen pressures for the hydrogen-water system were calculated from the relations

$$
\Delta F^{\circ}=-\mathrm{RT} \ln \mathrm{K}
$$

and

$$
\mathrm{K}=\mathrm{P}\left(\mathrm{H}_{2} \mathrm{O}\right) / \mathrm{P}\left(\mathrm{H}_{2}\right) \mathrm{P}\left(\mathrm{O}_{2}\right)^{1 / 2}
$$

Upon combining these equations and substituting $\log _{10} \mathrm{~N}$ (written as $\log N$ ) for natural logarithms, the following is obtained:

$$
\log \mathrm{P}\left(\mathrm{O}_{2}\right)=\frac{2 \Delta \mathrm{F}^{\circ}}{2.303 \mathrm{RT}}+2 \log \left[\frac{\mathrm{P}\left(\mathrm{H}_{2} \mathrm{O}\right)}{\mathrm{P}\left(\mathrm{H}_{2}\right)}\right] \text {, }
$$

where $R$, the gas constant, is taken as $1.987 \mathrm{cal} /{ }^{\circ} \mathrm{C}-m o l e$, and $\mathrm{T}$ is in ${ }^{\circ} \mathrm{K}$. The standard free energy for the formation of water is given by Kubaschewski and Evans (1) over the range from 298 to $2500^{\circ} \mathrm{K}$ by the equation

$$
\Delta F^{\circ}( \pm 1 \mathrm{kcal})=-58,900+13.1 \mathrm{~T}
$$

Values for $\triangle F^{\circ}$ are also given by Coughlin(2) at selected temperature points in the interval from 298.16 to $2000^{\circ} \mathrm{K}$. The oxygen pressures listed in Table I are based on Kubaschewski and Evans' equation, but they agree satisfactorily with values calculated from Coughlin's free energies. The water vapor pressures given in Table I correspond to three convenient temperature points: (a) 0.00053 Torr is the pressure above ice at $-78.5^{\circ} \mathrm{C}$ [Dushman(3)], the sublimation point of solid $\mathrm{CO}_{2} ;(\mathrm{b}) 4.6$ Torr is the pressure above water at $0^{\circ} \mathrm{C}$, and (c) 12.8 Torr is the aqueous pressure at $15^{\circ} \mathrm{C}$. 
Table I

PARTIAL PRESSURE OF OXYGEN IN EQUILIBRIUM WITH HYDROGEN AND WATER VAPOR*

\begin{tabular}{|c|c|c|c|c|c|c|c|c|c|c|}
\hline \multirow{2}{*}{$\begin{array}{l}\mathrm{P}\left(\mathrm{H}_{2}\right) \\
\text { Torr }\end{array}$} & \multirow{2}{*}{$\begin{array}{l}\mathrm{P}\left(\mathrm{H}_{2} \mathrm{O}\right) \\
\text { Torr }\end{array}$} & \multicolumn{9}{|c|}{$\mathrm{P}\left(\mathrm{O}_{2}\right)^{* *}(\mathrm{~atm})$ at the Listed Temperatures $\left({ }^{\circ} \mathrm{C}\right)$} \\
\hline & & 600 & 625 & 650 & 675 & 700 & 785 & 750 & 775 & 800 \\
\hline 800 & $\begin{array}{r}46 \\
128\end{array}$ & $\begin{array}{l}61(-29) \\
48(-28)\end{array}$ & $\begin{array}{ll}4 & 0(-28) \\
32 & (-27)\end{array}$ & $\begin{array}{l}24(-27) \\
19(-26)\end{array}$ & $\begin{array}{l}13(-26) \\
10(-25)\end{array}$ & $\begin{array}{l}65(-26) \\
51(-25)\end{array}$ & $\begin{array}{l}30(-25) \\
23(-24)\end{array}$ & $\begin{array}{l}13(-24) \\
10(-23)\end{array}$ & $\begin{array}{l}51(-24) \\
40(-23)\end{array}$ & $\begin{array}{l}19(-23) \\
15(-22)\end{array}$ \\
\hline 400 & $\begin{array}{r}46 \\
128\end{array}$ & $\begin{array}{l}24(-28) \\
19(-27)\end{array}$ & $\begin{array}{l}16(-27) \\
13(-26)\end{array}$ & $\begin{array}{l}96(-27) \\
75(-26)\end{array}$ & $\begin{array}{l}52(-26) \\
41(-25)\end{array}$ & $\begin{array}{l}26(-25) \\
20(-24)\end{array}$ & $\begin{array}{l}12(-24) \\
94(-24)\end{array}$ & $\begin{array}{l}51(-24) \\
40(-23)\end{array}$ & $\begin{array}{l}20(-23) \\
16(-22)\end{array}$ & $\begin{array}{l}76(-23) \\
60(-22)\end{array}$ \\
\hline 200 & $\begin{array}{r}46 \\
128\end{array}$ & $\begin{array}{l}98(-28) \\
76(-27)\end{array}$ & $\begin{array}{l}65(-27) \\
50(-26)\end{array}$ & $\begin{array}{l}39(-26) \\
30(-25)\end{array}$ & $\begin{array}{l}21(-25) \\
16(-24)\end{array}$ & $\begin{array}{l}10(-24) \\
82(-24)\end{array}$ & $\begin{array}{l}48(-24) \\
37(-23)\end{array}$ & $\begin{array}{l}20(-23) \\
16(-22)\end{array}$ & $\begin{array}{l}81(-23) \\
64(-22)\end{array}$ & $\begin{array}{l}30(-22) \\
24(-21)\end{array}$ \\
\hline 80 & $\begin{array}{r}46 \\
128\end{array}$ & $\begin{array}{l}61(-27) \\
48(-26)\end{array}$ & $\begin{array}{l}40(-26) \\
32(-25)\end{array}$ & $\begin{array}{l}24(-25) \\
19(-24)\end{array}$ & $\begin{array}{l}13(-24) \\
10(-23)\end{array}$ & $\begin{array}{l}65(-24) \\
51(-23)\end{array}$ & $\begin{array}{l}30(-23) \\
23(-22)\end{array}$ & $\begin{array}{l}13(-22) \\
10(-21)\end{array}$ & $\begin{array}{l}51(-22) \\
40(-21)\end{array}$ & $\begin{array}{l}19(-21) \\
15(-20)\end{array}$ \\
\hline 40 & $\begin{array}{r}46 \\
128\end{array}$ & $\begin{array}{l}24(-26) \\
19(-25)\end{array}$ & $\begin{array}{l}16(-25) \\
13(-24)\end{array}$ & $\begin{array}{l}96(-25) \\
75(-24)\end{array}$ & $\begin{array}{l}52(-24) \\
41(-23)\end{array}$ & $\begin{array}{l}26(-23) \\
20(-22)\end{array}$ & $\begin{array}{l}12(-22) \\
94(-22)\end{array}$ & $\begin{array}{l}51(-22) \\
40(-21)\end{array}$ & $\begin{array}{l}20(-21) \\
16(-20)\end{array}$ & $\begin{array}{l}76(-21) \\
59(-20)\end{array}$ \\
\hline 20 & $\begin{array}{r}46 \\
128\end{array}$ & $\begin{array}{l}98(-26) \\
76(-25)\end{array}$ & $\begin{array}{l}65(-25) \\
50(-24)\end{array}$ & $\begin{array}{l}39(-24) \\
30(-23)\end{array}$ & $\begin{array}{l}21(-23) \\
16(-22)\end{array}$ & $\begin{array}{l}10(-22) \\
82(-22)\end{array}$ & $\begin{array}{l}48(-22) \\
37(-21)\end{array}$ & $\begin{array}{l}20(-21) \\
16(-20)\end{array}$ & $\begin{array}{l}81(-21) \\
64(-20)\end{array}$ & $\begin{array}{l}30(-20) \\
24(-19)\end{array}$ \\
\hline 8 & $\begin{array}{r}46 \\
128\end{array}$ & $\begin{array}{l}61(-25) \\
48(-24)\end{array}$ & $\begin{array}{l}40(-24) \\
32(-23)\end{array}$ & $\begin{array}{l}24(-23) \\
19(-22)\end{array}$ & $\begin{array}{l}13(-22) \\
10(-21)\end{array}$ & $\begin{array}{l}65(-22) \\
51(-21)\end{array}$ & $\begin{array}{l}30(-21) \\
23(-20)\end{array}$ & $\begin{array}{l}13(-20) \\
11(-19)\end{array}$ & $\begin{array}{l}51(-20) \\
40(-19)\end{array}$ & $\begin{array}{l}19(-19) \\
15(-18)\end{array}$ \\
\hline 4 & $\begin{array}{r}46 \\
128\end{array}$ & $\begin{array}{l}24(-24) \\
19(-23)\end{array}$ & $\begin{array}{l}16(-23) \\
13(-22)\end{array}$ & $\begin{array}{l}96(-23) \\
75(-22)\end{array}$ & $\begin{array}{l}52(-22) \\
41(-21)\end{array}$ & $\begin{array}{l}26(-21) \\
20(-20)\end{array}$ & $\begin{array}{l}12(-20) \\
94(-20)\end{array}$ & $\begin{array}{l}51(-20) \\
40(-19)\end{array}$ & $\begin{array}{ll}20 & (-19) \\
16 & (-18)\end{array}$ & $\begin{array}{l}76(-19) \\
59(-18)\end{array}$ \\
\hline
\end{tabular}

\begin{tabular}{|c|c|c|c|c|c|c|c|c|c|c|}
\hline & & 825 & 850 & 875 & 900 & 925 & 950 & 975 & 1000 & 1025 \\
\hline 800 & $\begin{array}{c}000053 \\
46 \\
128\end{array}$ & $\begin{array}{l}90(-31) \\
67(-23) \\
52(-22)\end{array}$ & $\begin{array}{ll}29 & (-30) \\
2 & 2(-22) \\
1 & 7(-21)\end{array}$ & $\begin{array}{l}94(-30) \\
70(-22) \\
54(-21)\end{array}$ & $\begin{array}{l}28(-29) \\
21(-21) \\
16(-20)\end{array}$ & $\begin{array}{l}80(29) \\
60(-21) \\
47(-20)\end{array}$ & $\begin{array}{l}21(-28) \\
16(-20) \\
13(-19)\end{array}$ & $\begin{array}{l}59(-28) \\
44(-20) \\
34(-19)\end{array}$ & $\begin{array}{l}15(-27) \\
11(-19) \\
86(-19)\end{array}$ & 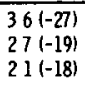 \\
\hline 400 & $\begin{array}{l}000053 \\
46 \\
128\end{array}$ & $\begin{array}{l}36(-30) \\
27(-22) \\
21(-21)\end{array}$ & $\begin{array}{ll}1 & 2(-29) \\
8 & 9(-22) \\
6 & 9(-21)\end{array}$ & $\begin{array}{l}38(-29) \\
28(-21) \\
22(-20)\end{array}$ & $\begin{array}{l}11(-28) \\
84(-21) \\
66(-20)\end{array}$ & $\begin{array}{l}32(-28) \\
24(-20) \\
19(-19)\end{array}$ & $\begin{array}{l}89(-28) \\
66(-20) \\
52(-19)\end{array}$ & $\begin{array}{l}23(-27) \\
17(-19) \\
14(-18)\end{array}$ & $\begin{array}{l}59(-27) \\
44(-19) \\
35(-18)\end{array}$ & $\begin{array}{l}15(-26) \\
11(-18) \\
85(-18)\end{array}$ \\
\hline 200 & $\begin{array}{c}000053 \\
46 \\
128\end{array}$ & $\begin{array}{l}15(-29) \\
11(-21) \\
83(-21)\end{array}$ & $\begin{array}{l}47(-29) \\
35(-21) \\
28(-20)\end{array}$ & $\begin{array}{l}15(-28) \\
11(-20) \\
87(-20)\end{array}$ & $\begin{array}{l}46(-28) \\
34(-20) \\
26(-19)\end{array}$ & $\begin{array}{l}13(-27) \\
96(-20) \\
75(-19)\end{array}$ & $\begin{array}{l}35(-27) \\
26(-19) \\
21(-18)\end{array}$ & $\begin{array}{l}94(-27) \\
70(-19) \\
54(-18)\end{array}$ & $\begin{array}{l}24(-26) \\
18(-18) \\
14(-17)\end{array}$ & $\begin{array}{l}58(-26) \\
43(-18) \\
34(-17)\end{array}$ \\
\hline 80 & $\begin{array}{c}000053 \\
46 \\
128\end{array}$ & $\begin{array}{l}90(-29) \\
67(-21) \\
52(-20)\end{array}$ & $\begin{array}{l}29(-28) \\
22(-20) \\
17(-19)\end{array}$ & $\begin{array}{l}94(-28) \\
70(-20) \\
54(-19)\end{array}$ & $\begin{array}{l}28(-27) \\
21(-19) \\
16(-18)\end{array}$ & $\begin{array}{l}80(-27) \\
60(-19) \\
47(18)\end{array}$ & $\begin{array}{l}21(-26) \\
16(-18) \\
13(-17)\end{array}$ & $\begin{array}{l}59(-26) \\
44(-18) \\
34(-17)\end{array}$ & $\begin{array}{l}15(-25) \\
11(-17) \\
86(-17)\end{array}$ & $\begin{array}{l}36(-25) \\
27(-17) \\
21(-16)\end{array}$ \\
\hline 40 & $\begin{array}{c}000053 \\
46 \\
128\end{array}$ & $\begin{array}{l}36(-28) \\
27(-20) \\
21(-19)\end{array}$ & $\begin{array}{l}12(-27) \\
89(-20) \\
69(-19)\end{array}$ & $\begin{array}{l}38(-27) \\
28(-19) \\
22(-18)\end{array}$ & $\begin{array}{l}11(-26) \\
84(-19) \\
66(-18)\end{array}$ & $\begin{array}{l}32(-26) \\
24(-18) \\
19(-17)\end{array}$ & $\begin{array}{l}89(-26) \\
66(-18) \\
52(-17)\end{array}$ & $\begin{array}{l}23(-25) \\
17(-17) \\
14(-16)\end{array}$ & $\begin{array}{l}59(-25) \\
44(-17) \\
35(-16)\end{array}$ & $\begin{array}{l}15(-24) \\
11(16) \\
85(-16)\end{array}$ \\
\hline 20 & $\begin{array}{l}000053 \\
46 \\
128\end{array}$ & $\begin{array}{l}15(-27) \\
11(-19) \\
83(-19)\end{array}$ & $\begin{array}{l}47(-27) \\
35(-19) \\
28(-18)\end{array}$ & $\begin{array}{l}15(-26) \\
11(-18) \\
87(-18)\end{array}$ & $\begin{array}{l}46(-26) \\
34(-18) \\
26(-17)\end{array}$ & $\begin{array}{l}13(-25) \\
96(-18) \\
75(-17)\end{array}$ & $\begin{array}{l}35(25) \\
26(-17) \\
21(-16)\end{array}$ & $\begin{array}{ll}9 & 4(-25) \\
70 & (-17) \\
5 & 4(-16)\end{array}$ & $\begin{array}{l}24(-24) \\
18(-16) \\
14(-15)\end{array}$ & $\begin{array}{l}58(-24) \\
43(-16) \\
34(-15)\end{array}$ \\
\hline 8 & $\begin{array}{l}000053 \\
46 \\
128\end{array}$ & $\begin{array}{ll}9 & 0(-27) \\
6 & 7(-19) \\
5 & 2(-18)\end{array}$ & $\begin{array}{l}29(-26) \\
22(-18) \\
17(-17)\end{array}$ & $\begin{array}{l}94(-26) \\
70(-18) \\
54(-17)\end{array}$ & $\begin{array}{l}28(-25) \\
21(-17) \\
16(-16)\end{array}$ & $\begin{array}{lll}8 & 0 & (-25) \\
6 & 0 & (-17) \\
4 & 7 & (-16)\end{array}$ & $\begin{array}{l}21(-24) \\
16(-16) \\
13(-15)\end{array}$ & $\begin{array}{l}59(-24) \\
44(-16) \\
34(-15)\end{array}$ & $\begin{array}{l}15(-23) \\
11(-15) \\
86(-15)\end{array}$ & $\begin{array}{l}36(-23) \\
27(-15) \\
21(-14)\end{array}$ \\
\hline 4 & $\begin{array}{l}000053 \\
46 \\
128\end{array}$ & $\begin{array}{l}36(-26) \\
27(-18) \\
21(-17)\end{array}$ & $\begin{array}{l}12(-25) \\
89(-18) \\
69(-17)\end{array}$ & $\begin{array}{l}38(-25) \\
28(-17) \\
22(16)\end{array}$ & $\begin{array}{l}11(-24) \\
84(-17) \\
66(-16)\end{array}$ & $\begin{array}{l}32(-24) \\
24(16) \\
19(-15)\end{array}$ & $\begin{array}{ll}8 & 9(-24) \\
6 & 6(-16) \\
5 & 2(-15)\end{array}$ & $\begin{array}{l}23(-23) \\
17(-15) \\
14(-14)\end{array}$ & $\begin{array}{l}59(-23) \\
44(-15) \\
35(-14)\end{array}$ & $\begin{array}{l}15(-22) \\
11(-14) \\
85(-14)\end{array}$ \\
\hline
\end{tabular}

\begin{tabular}{|c|c|c|c|c|c|c|c|c|c|c|}
\hline & & 1050 & 1075 & 1100 & 1125 & 1150 & 1175 & 1200 & 1225 & 1250 \\
\hline 800 & $\begin{array}{c}000053 \\
46 \\
128\end{array}$ & $\begin{array}{l}85(-27) \\
64(-19) \\
50(-18)\end{array}$ & $\begin{array}{l}15(-26) \\
11(-18) \\
87(-18)\end{array}$ & $\begin{array}{l}44(26) \\
33(-18) \\
26(-17)\end{array}$ & 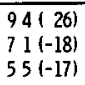 & $\begin{array}{l}20(-25) \\
15(-17) \\
12(-16)\end{array}$ & $\begin{array}{l}41(-25) \\
31(17) \\
24(-16)\end{array}$ & $\begin{array}{l}81(-25) \\
61(-17) \\
48(-16)\end{array}$ & 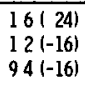 & $\begin{array}{ll}3 & 1(-24) \\
2 & 3(-16) \\
1 & 8(-15)\end{array}$ \\
\hline 400 & $\begin{array}{c}000053 \\
46 \\
128\end{array}$ & $\begin{array}{ll}3 & 5(-26) \\
2 & 6(18) \\
2 & 0(-17)\end{array}$ & $\begin{array}{l}60(-26) \\
45(-18) \\
35(-17)\end{array}$ & $\begin{array}{l}17(-8) \\
13(17) \\
10(-16)\end{array}$ & $\begin{array}{l}37(-25) \\
28(-17) \\
22(-16)\end{array}$ & $\begin{array}{l}80(25) \\
60(-17) \\
47(-16)\end{array}$ & $\begin{array}{l}16(-24) \\
12(16) \\
96(-16)\end{array}$ & $\begin{array}{l}32(-24) \\
24(-16) \\
19(-15)\end{array}$ & $\begin{array}{l}64(-24) \\
48(-16) \\
37(-15)\end{array}$ & $\begin{array}{l}12(23) \\
92(-16) \\
72(-15)\end{array}$ \\
\hline 200 & $\begin{array}{l}000053 \\
46 \\
128\end{array}$ & $\begin{array}{lll}1 & 3 & (-25) \\
1 & 0(-17) \\
8 & 0(-17)\end{array}$ & $\begin{array}{l}24(-25) \\
18(-17) \\
14(-16)\end{array}$ & $\begin{array}{l}69(25) \\
52(-17) \\
41(-16)\end{array}$ & $\begin{array}{l}15(-24) \\
11(-16) \\
89(-16)\end{array}$ & $\begin{array}{l}32(-24) \\
24(-16) \\
19(-15)\end{array}$ & $\begin{array}{l}65(-24) \\
49(-16) \\
38(-15)\end{array}$ & $\begin{array}{l}13(-23) \\
98(-16) \\
76(-15)\end{array}$ & $\begin{array}{l}25(-23) \\
19(-15) \\
15(-14)\end{array}$ & $\begin{array}{l}49(-23) \\
37(-15) \\
29(-14)\end{array}$ \\
\hline 80 & $\begin{array}{l}000053 \\
46 \\
128\end{array}$ & $\begin{array}{lll}8 & 5(-25) \\
6 & 4(-17) \\
5 & 0 & (-16)\end{array}$ & $\begin{array}{l}15(-24) \\
11(-16) \\
87(-16)\end{array}$ & $\begin{array}{l}44(-24) \\
33(-16) \\
26(-15)\end{array}$ & $\begin{array}{l}94(-24) \\
71(-16) \\
55(-15)\end{array}$ & $\begin{array}{l}20(-23) \\
15(-15) \\
12(-14)\end{array}$ & $\begin{array}{l}41(-23) \\
31(-15) \\
24(-14)\end{array}$ & $\begin{array}{l}81(-23) \\
61(-15) \\
48(-14)\end{array}$ & $\begin{array}{l}16(-22) \\
12(-14) \\
94(-14)\end{array}$ & $\begin{array}{l}31(-22) \\
23(-14) \\
18(-13)\end{array}$ \\
\hline 40 & $\begin{array}{l}000053 \\
46 \\
128\end{array}$ & $\begin{array}{l}35(-24) \\
26(-16) \\
20(-15)\end{array}$ & $\begin{array}{l}60(-24) \\
45(-16) \\
35(-15)\end{array}$ & $\begin{array}{l}17(-23) \\
13(-15) \\
10(14)\end{array}$ & $\begin{array}{l}37(-23) \\
28(-15) \\
22(-14)\end{array}$ & $\begin{array}{l}80(-23) \\
58(-15) \\
47(-14)\end{array}$ & $\begin{array}{l}16(-22) \\
12(-14) \\
96(-14)\end{array}$ & $\begin{array}{l}32(-22) \\
24(-14) \\
19(-13)\end{array}$ & $\begin{array}{l}64(-22) \\
48(-14) \\
37(-13)\end{array}$ & $\begin{array}{l}12(-21) \\
92(-14) \\
72(-13)\end{array}$ \\
\hline 20 & $\begin{array}{l}000053 \\
46 \\
128\end{array}$ & $\begin{array}{ll}13 & (-23) \\
10 & 0(-15) \\
80 & (-15)\end{array}$ & $\begin{array}{ll}24 & (-23) \\
1 & (-15) \\
1 & (-14)\end{array}$ & $\begin{array}{l}69(-23) \\
52(-15) \\
41(-14)\end{array}$ & $\begin{array}{lll}1 & 5 & (-22) \\
1 & 1 & (-14) \\
8 & 9 & (-14)\end{array}$ & $\begin{array}{l}32(22) \\
24(-14) \\
19 \quad 13)\end{array}$ & $\begin{array}{l}65(22) \\
49(-14) \\
38(-13)\end{array}$ & $\begin{array}{l}13(-21) \\
98(-14) \\
76(-13)\end{array}$ & $\begin{array}{l}25(-21) \\
19(-13) \\
15(-12)\end{array}$ & $\begin{array}{l}49(-21) \\
37(-13) \\
29(-12)\end{array}$ \\
\hline 8 & $\begin{array}{c}000053 \\
46 \\
128\end{array}$ & $\begin{array}{ll}85 & (-23) \\
64 & (-15) \\
50 & (-14)\end{array}$ & $\begin{array}{l}15(-22) \\
11(-14) \\
87(-14)\end{array}$ & $\begin{array}{l}44(-22) \\
33(-14) \\
26(-13)\end{array}$ & $\begin{array}{l}94(-22) \\
71(-14) \\
55(-13)\end{array}$ & $\begin{array}{l}20(-21) \\
15(-13) \\
12(-12)\end{array}$ & $\begin{array}{l}41(-21) \\
31(-13) \\
24(-12)\end{array}$ & $\begin{array}{l}81(-21) \\
61(-13) \\
48(-12)\end{array}$ & $\begin{array}{l}16(-20) \\
12(-12) \\
94(-12)\end{array}$ & $\begin{array}{l}31(-20) \\
23(-12) \\
18(-11)\end{array}$ \\
\hline 4 & $\begin{array}{l}000053 \\
46 \\
128\end{array}$ & $\begin{array}{l}35(-22) \\
26(-14) \\
20(-13)\end{array}$ & $\begin{array}{l}60(-22) \\
45(-14) \\
35(-13)\end{array}$ & $\begin{array}{l}17(-21) \\
13(-13) \\
10(-12)\end{array}$ & $\begin{array}{l}37(-21) \\
28(-13) \\
22(-12)\end{array}$ & $\begin{array}{l}80(-21) \\
58(-13) \\
47(-12)\end{array}$ & $\begin{array}{ll}1 & 6(-20) \\
1 & 2(-12) \\
9 & 6(-12)\end{array}$ & $\begin{array}{l}32(-20) \\
24(-12) \\
19(-11)\end{array}$ & $\begin{array}{l}64(-20) \\
48(-12) \\
37(-11)\end{array}$ & $\begin{array}{ll}1 & 2(-19) \\
9 & 2(-12) \\
7 & 2(-11)\end{array}$ \\
\hline
\end{tabular}

- $\mathrm{P}\left(\mathrm{O}_{2}\right)$ may be calculated for interpolated or extrapolated values of $\mathrm{P}\left(\mathrm{H}_{2} \mathrm{O}\right)$ or $\mathrm{P}\left(\mathrm{H}_{2}\right)$ at constant temperature with the relations

$$
P_{1}\left(O_{2}\right)=P_{2}\left(O_{2}\right)\left[\frac{P_{1}\left(H_{2} O\right)}{P_{2}\left(\mathrm{H}_{2} O\right)}\right]^{2} \quad P_{1}\left(O_{2}\right)=P_{2}\left(O_{2}\right)\left[\frac{P_{2}\left(H_{2}\right)}{P_{1}\left(H_{2}\right)}\right]^{2}
$$

* The term $61(-29)$ should be read as $61 \times 10^{-29} \mathrm{~atm}$ etc 
Table I Cont'd

\begin{tabular}{|c|c|c|c|c|c|c|c|c|c|c|}
\hline \multirow{2}{*}{$\begin{array}{l}\mathrm{P}\left(\mathrm{H}_{2}\right) \\
\text { Torr }\end{array}$} & \multirow{2}{*}{$\begin{array}{c}\mathrm{P}\left(\mathrm{H}_{2} \mathrm{O}\right) \\
\text { Torr }\end{array}$} & \multicolumn{9}{|c|}{$\mathrm{P}\left(\mathrm{O}_{2}\right)(\mathrm{atm})$ at the Listed Temperatures $\left({ }^{\circ} \mathrm{C}\right)$} \\
\hline & & 1275 & 1300 & 1325 & 1350 & 1375 & 1400 & 1425 & 1450 & 1475 \\
\hline 800 & $\begin{array}{l}000053 \\
46 \\
128\end{array}$ & $\begin{array}{l}57(-24) \\
43(-16) \\
34(-15)\end{array}$ & $\begin{array}{l}10(-23) \\
79(-16) \\
62(-15)\end{array}$ & $\begin{array}{l}19(-23) \\
14(-15) \\
11(-14)\end{array}$ & $\begin{array}{l}33(-23) \\
25(-15) \\
20(-14)\end{array}$ & $\begin{array}{l}58(-23) \\
44(-15) \\
34(-14)\end{array}$ & $\begin{array}{l}10(-22) \\
75(-15) \\
59(-14)\end{array}$ & $\begin{array}{l}17(-22) \\
13(-14) \\
99(-14)\end{array}$ & $\begin{array}{l}28(-22) \\
21(-14) \\
16(-13)\end{array}$ & $\begin{array}{l}45(-22) \\
34(-14) \\
27(-13)\end{array}$ \\
\hline 400 & $\begin{array}{l}000053 \\
46 \\
128\end{array}$ & $\begin{array}{l}23(-23) \\
17(-15) \\
13(-14)\end{array}$ & $\begin{array}{l}43(-23) \\
32(-15) \\
25(-14)\end{array}$ & $\begin{array}{l}76(-23) \\
57(-15) \\
44(-14)\end{array}$ & $\begin{array}{l}13(-22) \\
10(-14) \\
79(-14)\end{array}$ & $\begin{array}{l}23(-22) \\
17(-14) \\
14(-13)\end{array}$ & $\begin{array}{l}40(22) \\
30(-14) \\
23(-13)\end{array}$ & $\begin{array}{l}66(-22) \\
50(-14) \\
39(-13)\end{array}$ & $\begin{array}{l}11(-21) \\
84(14) \\
65(-13)\end{array}$ & $\begin{array}{l}I 9(-21) \\
14(13) \\
1 I(-12)\end{array}$ \\
\hline 200 & $\begin{array}{l}000053 \\
46 \\
128\end{array}$ & $\begin{array}{l}92(-23) \\
69(-15) \\
54(-14)\end{array}$ & $\begin{array}{l}17(-22) \\
13(-14) \\
99(-14)\end{array}$ & $\begin{array}{l}31(-22) \\
23(-14) \\
18(-13)\end{array}$ & $\begin{array}{l}53(-22) \\
40(-14) \\
31(-13)\end{array}$ & $\begin{array}{l}93(-22) \\
70(-14) \\
55(-13)\end{array}$ & $\begin{array}{l}16(-21) \\
12(-13) \\
94(13)\end{array}$ & $\begin{array}{l}27(-21) \\
20(-13) \\
16(-12)\end{array}$ & $\begin{array}{l}44(-21) \\
33(-13) \\
26(-12)\end{array}$ & $\begin{array}{l}73(-21) \\
55(-13) \\
43(12)\end{array}$ \\
\hline 80 & $\begin{array}{l}000053 \\
46 \\
128\end{array}$ & $\begin{array}{l}57(-22) \\
43(-14) \\
34(-13)\end{array}$ & $\begin{array}{l}10(-21) \\
79(-14) \\
62(-13)\end{array}$ & $\begin{array}{l}19(-21) \\
14(-13) \\
11(-12)\end{array}$ & $\begin{array}{l}33(-21) \\
25(-13) \\
20(-12)\end{array}$ & $\begin{array}{l}58(-21) \\
44(-13) \\
34(-12)\end{array}$ & $\begin{array}{l}10(-20) \\
75(-13) \\
59(-12)\end{array}$ & $\begin{array}{l}17(-20) \\
13(-12) \\
99(-12)\end{array}$ & $\begin{array}{l}28(-20) \\
21(-12) \\
16(-11)\end{array}$ & $\begin{array}{l}45(-20) \\
34(12) \\
27(-11)\end{array}$ \\
\hline 40 & $\begin{array}{l}000053 \\
46 \\
128\end{array}$ & $\begin{array}{l}23(-21) \\
17(-13) \\
13(-12)\end{array}$ & $\begin{array}{l}43(-21) \\
32(-13) \\
25(-12)\end{array}$ & $\begin{array}{l}76(-21) \\
57(-13) \\
44(-12)\end{array}$ & $\begin{array}{l}13(-20) \\
10(-12) \\
79(-12)\end{array}$ & $\begin{array}{l}23(-20) \\
17(-12) \\
14(-11)\end{array}$ & $\begin{array}{l}40(-20) \\
30(-12) \\
23(-11)\end{array}$ & $\begin{array}{l}66(-20) \\
50(-12) \\
39(-11)\end{array}$ & $\begin{array}{l}11(19) \\
84(-12) \\
65(-11)\end{array}$ & $\begin{array}{l}19(-19) \\
14(-11) \\
11(-10)\end{array}$ \\
\hline 20 & $\begin{array}{l}000053 \\
46 \\
128\end{array}$ & $\begin{array}{l}92(-21) \\
69(-13) \\
54(-12)\end{array}$ & $\begin{array}{l}17(-20) \\
13(-12) \\
99(-12)\end{array}$ & $\begin{array}{l}31(-20) \\
23(-12) \\
18(-11)\end{array}$ & $\begin{array}{l}53(-20) \\
40(-12) \\
31(-11)\end{array}$ & $\begin{array}{l}93(-20) \\
70(-12) \\
55(-11)\end{array}$ & $\begin{array}{l}16(-19) \\
12(11) \\
94(-11)\end{array}$ & $\begin{array}{l}27(-19) \\
20(-11) \\
16(-10)\end{array}$ & $\begin{array}{l}44(-19) \\
33(-11) \\
26(-10)\end{array}$ & $\begin{array}{l}73(19) \\
55(-11) \\
43(10)\end{array}$ \\
\hline 8 & $\begin{array}{l}000053 \\
46 \\
128\end{array}$ & $\begin{array}{l}57(-20) \\
43(-12) \\
34(-11)\end{array}$ & $\begin{array}{l}10(-19) \\
79(-12) \\
62(-11)\end{array}$ & $\begin{array}{l}19(-19) \\
14(-11) \\
11(-10)\end{array}$ & $\begin{array}{l}33(-19) \\
25(-11) \\
20(-10)\end{array}$ & $\begin{array}{l}58(-19) \\
44(-11) \\
34(-10)\end{array}$ & $\begin{array}{l}10(-18) \\
75(-11) \\
59(-10)\end{array}$ & $\begin{array}{l}17(-18) \\
13(-10) \\
99(-10)\end{array}$ & $\begin{array}{l}28(-18) \\
21(-10) \\
16(-9)\end{array}$ & $\begin{array}{l}45(-18) \\
34(-10) \\
27(-9)\end{array}$ \\
\hline 4 & $\begin{array}{l}000053 \\
46 \\
128\end{array}$ & $\begin{array}{l}23(-19) \\
17(-11) \\
13(-10)\end{array}$ & $\begin{array}{l}43(-19) \\
32(-11) \\
25(-10)\end{array}$ & $\begin{array}{l}76(-19) \\
57(-11) \\
44(-10)\end{array}$ & $\begin{array}{l}13(-18) \\
10(-10) \\
79(-10)\end{array}$ & $\begin{array}{l}23(-18) \\
17(-10) \\
14(-9)\end{array}$ & $\begin{array}{l}40(-18) \\
30(-10) \\
23(-9)\end{array}$ & $\begin{array}{l}66(-18) \\
50(-10) \\
39(-9)\end{array}$ & $\begin{array}{l}11(-17) \\
84(-10) \\
65(-9)\end{array}$ & $\begin{array}{l}19(-17) \\
14(-9) \\
11(8)\end{array}$ \\
\hline & & 1500 & 1525 & 1550 & 1575 & 1600 & 1625 & 1650 & 1675 & 1700 \\
\hline 800 & $\begin{array}{l}000053 \\
46 \\
128\end{array}$ & $\begin{array}{l}73(-22) \\
55(-14) \\
43(-13)\end{array}$ & $\begin{array}{l}12(-21) \\
88(-14) \\
68(-13)\end{array}$ & $\begin{array}{l}19(-21) \\
14(-13) \\
11(-12)\end{array}$ & $\begin{array}{l}28(-21) \\
21(-13) \\
17(-12)\end{array}$ & $\begin{array}{l}44(-21) \\
33(-13) \\
26(-12)\end{array}$ & $\begin{array}{l}66(-21) \\
50(-13) \\
39(-12)\end{array}$ & $\begin{array}{l}10(-20) \\
75(-13) \\
58(-12)\end{array}$ & $\begin{array}{l}15(-20) \\
11(-12) \\
87(-12)\end{array}$ & $\begin{array}{l}21(-20) \\
16(-12) \\
13(-11)\end{array}$ \\
\hline 400 & $\begin{array}{l}000053 \\
46 \\
128\end{array}$ & $\begin{array}{l}29(-21) \\
22(-13) \\
17(-12)\end{array}$ & $\begin{array}{l}47(-21) \\
35(-13) \\
27(-12)\end{array}$ & $\begin{array}{l}73(-21) \\
55(-13) \\
43(-12)\end{array}$ & $\begin{array}{l}11(-20) \\
86(-13) \\
66(-12)\end{array}$ & $\begin{array}{l}17(-20) \\
13(-12) \\
10(-11)\end{array}$ & $\begin{array}{l}27(-20) \\
20(-12) \\
16(-11)\end{array}$ & $\begin{array}{l}40(-20) \\
30(-12) \\
23(-11)\end{array}$ & $\begin{array}{l}60(-20) \\
45(-12) \\
35(-11)\end{array}$ & $\begin{array}{l}86(-20) \\
65(-12) \\
51(-11)\end{array}$ \\
\hline 200 & $\begin{array}{l}000053 \\
46 \\
128\end{array}$ & $\begin{array}{l}12(-20) \\
88(-13) \\
69(-12)\end{array}$ & $\begin{array}{l}19(-20) \\
14(-12) \\
11(-11)\end{array}$ & $\begin{array}{l}29(-20) \\
22(-12) \\
17(-11)\end{array}$ & $\begin{array}{l}45(-20) \\
34(-12) \\
27(-11)\end{array}$ & $\begin{array}{l}70(-20) \\
53(-12) \\
41(-11)\end{array}$ & $\begin{array}{l}11(-19) \\
80(-12) \\
62(-11)\end{array}$ & $\begin{array}{l}16(-19) \\
12(-11) \\
93(-11)\end{array}$ & $\begin{array}{l}24(-19) \\
18(-1) \\
14(-10)\end{array}$ & $\begin{array}{l}35(19) \\
26(-11) \\
20(-10)\end{array}$ \\
\hline 80 & $\begin{array}{l}000053 \\
46 \\
128\end{array}$ & $\begin{array}{l}73(-20) \\
55(-12) \\
43(-11)\end{array}$ & $\begin{array}{l}12(-19) \\
88(-12) \\
68(-11)\end{array}$ & $\begin{array}{l}19(-19) \\
14(-11) \\
11(-10)\end{array}$ & $\begin{array}{l}28(-19) \\
2 \geq(-11) \\
17(-10)\end{array}$ & $\begin{array}{l}44(-19) \\
33(-11) \\
26(-10)\end{array}$ & $\begin{array}{l}66(-19) \\
50(-11) \\
39(-10)\end{array}$ & $\begin{array}{l}10(-18) \\
75(-11) \\
58(-10)\end{array}$ & $\begin{array}{l}15(-18) \\
11(-10) \\
87(-10)\end{array}$ & $\begin{array}{lll}2 & 1 & (-18) \\
1 & 6 & (10) \\
1 & 3 & (-9)\end{array}$ \\
\hline 40 & $\begin{array}{l}000053 \\
46 \\
128\end{array}$ & $\begin{array}{l}29(-19) \\
22(-11) \\
17(-10)\end{array}$ & $\begin{array}{l}47(-19) \\
35(-11) \\
27(-10)\end{array}$ & $\begin{array}{l}73(-19) \\
55(-11) \\
43(-10)\end{array}$ & $\begin{array}{l}11(-18) \\
86(-11) \\
66(-10)\end{array}$ & $\begin{array}{l}17(-18) \\
13(-10) \\
10(-9)\end{array}$ & $\begin{array}{l}27(-18) \\
20(-10) \\
16(-9)\end{array}$ & $\begin{array}{l}40(-18) \\
30(-10) \\
23(9)\end{array}$ & $\begin{array}{l}60(-18) \\
45(-10) \\
35(9)\end{array}$ & $\begin{array}{l}86(-18) \\
65(-10) \\
51(-9)\end{array}$ \\
\hline 20 & $\begin{array}{l}000053 \\
46 \\
128\end{array}$ & $\begin{array}{l}12(-18) \\
88(-11) \\
69(-10)\end{array}$ & $\begin{array}{l}19(-18) \\
14(-10) \\
11(-9)\end{array}$ & $\begin{array}{l}29(-18) \\
22(-10) \\
17(-9)\end{array}$ & $\begin{array}{l}45(-18) \\
34(-10) \\
27(-9)\end{array}$ & $\begin{array}{l}70(-18) \\
53(-10) \\
41(-9)\end{array}$ & $\begin{array}{l}11(-17) \\
80(-10) \\
62(-9)\end{array}$ & $\begin{array}{l}16(-17) \\
12(-9) \\
93(-9)\end{array}$ & $\begin{array}{l}24(-17) \\
18(9) \\
14(-8)\end{array}$ & $\begin{array}{l}35(-17) \\
26(-9) \\
20(-8)\end{array}$ \\
\hline 8 & $\begin{array}{l}000053 \\
46 \\
128\end{array}$ & $\begin{array}{l}73(-18) \\
55(-10) \\
43(-9)\end{array}$ & $\begin{array}{l}12(-17) \\
88(-10) \\
68(-9)\end{array}$ & 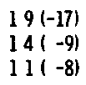 & $\begin{array}{l}28(-17) \\
21(-9) \\
17(-8)\end{array}$ & $\begin{array}{l}44(-17) \\
33(-9) \\
26(-8)\end{array}$ & $\begin{array}{l}66(-17) \\
50(-9) \\
39(-8)\end{array}$ & $\begin{array}{l}10(-16) \\
75(9) \\
58(-8)\end{array}$ & $\begin{array}{l}15(-16) \\
11(-8) \\
87(-8)\end{array}$ & $\begin{array}{l}21(-16) \\
16(-8) \\
13(-7)\end{array}$ \\
\hline 4 & $\begin{array}{l}000053 \\
46 \\
128\end{array}$ & $\begin{array}{l}29(-17) \\
22(-9) \\
17(-8)\end{array}$ & $\begin{array}{l}47(-17) \\
35(-9) \\
27(-8)\end{array}$ & $\begin{array}{l}73(-17) \\
55(-9) \\
43(-8)\end{array}$ & $\begin{array}{l}11(-16) \\
86(-9) \\
67(-8)\end{array}$ & $\begin{array}{l}17(-16) \\
13(-8) \\
10(-7)\end{array}$ & $\begin{array}{l}27(-16) \\
20(-8) \\
16(-7)\end{array}$ & $\begin{array}{l}40(-16) \\
30(-8) \\
23(-7)\end{array}$ & $\begin{array}{l}60(-16) \\
45(-8) \\
35(-7)\end{array}$ & $\begin{array}{l}86(-16) \\
65(-8) \\
51(7)\end{array}$ \\
\hline
\end{tabular}

DISSOCIATION OF CUPRIC OXIDE

Oxygen pressures were determined from the dissociation of $\mathrm{CuO}$ by the relation

$$
\Delta F^{\circ}=-\mathrm{RT} \ln \mathrm{P}\left(\mathrm{O}_{2}\right)^{1 / 2},
$$

which may be rearranged into the form

$$
\log P\left(\mathrm{O}_{2}\right)=-2 \Delta \mathrm{F}^{\circ} / 2.303 \mathrm{RT}
$$


Kubaschewski and Evans give $\Delta \mathrm{F}^{\circ}$ for this reaction between 298 and $1300^{\circ} \mathrm{K}$ as

$$
\Delta \mathrm{F}^{\circ}( \pm 0.5 \mathrm{kcal})=34,950+6.1 \mathrm{~T} \log \mathrm{T}-44.3 \mathrm{~T}
$$

Coughlin lists values of $\Delta \mathrm{F}^{\circ}$ for the formation of $\mathrm{Cu}_{2} \mathrm{O}$ and $\mathrm{CuO}$ at several points between 298.16 and $2000^{\circ} \mathrm{K}$. Combination of these free energies in the term

$$
\Delta \mathrm{F}^{\circ}\left(2 \mathrm{Cu}+1 / 2 \mathrm{O}_{2} \rightarrow \mathrm{Cu}_{2} \mathrm{O}\right)-2 \Delta \mathrm{F}^{\circ}\left(\mathrm{Cu}+1 / 2 \mathrm{O}_{2} \rightarrow \mathrm{CuO}\right)
$$

gives values for $\Delta F^{\circ}\left(2 \mathrm{CuO} \rightarrow \mathrm{Cu}_{2} \mathrm{O}+1 / 2 \mathrm{O}_{2}\right)$ which agree satisfactorily with those given by equation (8). It should be emphasized that these expressions for $\triangle \mathrm{F}^{\circ}$ apply only when an equilibrated mixture of $\mathrm{CuO}, \mathrm{Cu}_{2} \mathrm{O}$, and $\mathrm{O}_{2}$ is present in the system. When this condition is fulfilled, neither nonstoichiometric compositions nor the further dissociation of $\mathrm{Cu}_{2} \mathrm{O}$ can introduce an error.

The oxygen pressures calculated from equations (7) and (8) and listed in Table II have been confirmed by Berkowitz, (4) who analyzed the oxygen content of a helium stream which had been passed over heated $\mathrm{CuO} / \mathrm{Cu}_{2} \mathrm{O}$.

Table II

DISSOCIAT ION PRESSURE OF OXYGEN FROM CUPRIC OXIDE

\begin{tabular}{|c|c||c|c|}
\hline Temp $\left({ }^{\circ} \mathrm{C}\right)$ & $\mathrm{P}\left(\mathrm{O}_{2}\right)(\mathrm{atm})$ & $\mathrm{Temp}\left({ }^{\circ} \mathrm{C}\right)$ & $\mathrm{P}\left(\mathrm{O}_{2}\right)(\mathrm{atm})$ \\
\hline 200 & $8.6(-21)$ & 625 & $3.0(-6)$ \\
225 & $3.1(-19)$ & 650 & $8.0(-6)$ \\
250 & $8.0(-18)$ & 675 & $2.0(-5)$ \\
275 & $1.5(-16)$ & 700 & $5.0(-5)$ \\
300 & $2.2(-15)$ & 725 & $1.2(-4)$ \\
325 & $2.6(-14)$ & 750 & $2.5(-4)$ \\
350 & $2.5(-13)$ & 775 & $5.4(-4)$ \\
375 & $2.0(-12)$ & 800 & $1.1(-3)$ \\
400 & $1.3(-11)$ & 825 & $2.2(-3)$ \\
425 & $7.8(-11)$ & 850 & $4.2(-3)$ \\
450 & $4.1(-10)$ & 875 & $7.9(-3)$ \\
475 & $1.9(-9)$ & 900 & $1.4(-2)$ \\
500 & $7.9(-9)$ & 925 & $2.5(-2)$ \\
525 & $3.0(-8)$ & 950 & $4.4(-2)$ \\
550 & $1.1(-7)$ & 975 & $7.4(-2)$ \\
575 & $3.5(-7)$ & 1000 & $1.2(-1)$ \\
600 & $1.0(-6)$ & & \\
\hline
\end{tabular}




\section{REFERENCES}

1. O. Kubaschewski and E. Ll. Evans, Metallurgical Thermochemistry, Table E, pp. 336-343, Pergamon Press, New York (1958).

2. J. P. Coughlin, Contributions to the Data on Theoretical Metallurgy, XII, Heats and Free Energies of Formation of Inorganic Oxides, Bull. 542 Bureau of Mines (1954) pp. 20 and 23.

3. S. Dushman, Scientific Foundations of Vacuum Technique, Second Edition, J. M. Lafferty, Ed., p. 728, John Wiley and Sons, New York (1962).

4. J. Berkowitz, Stability of Ceramic Materials at Temperatures to 2000ㄷ, WADD Tech. Rep. 60-377, pp. 4-5 (Sept 1960). 